\title{
Mid-range Wireless Energy Transfer Using Inductive Resonance for Wireless Sensors
}

\author{
Shahrzad Jalali Mazlouman, Alireza Mahanfar, Bozena Kaminska, \\ Simon Fraser University \\ \{sja53,nima_mahanfar, kaminska\}@sfu.ca
}

\begin{abstract}
Methods are suggested and tested to measure and optimize the wireless energy transfer efficiency for mid-range $(10-100 \mathrm{~cm})$ inductive coils with relatively low profile using magnetic resonance. These coils can be used to provide energy for wireless sensors and battery-operated devices. It is shown that for every system, a resonance frequency can be identified where the wireless energy transfer efficiency is optimal. Several prototypes are developed and tested as a proof of validity of the proposed technique. It is also shown that by tuning to the optimum resonant frequency and designing proper matching circuitry, an efficiency of about $25 \%$ for moderate profiles can be achieved.
\end{abstract}

\section{INTRODUCTION}

Wireless energy transfer methods based on near-field coupling $(D<\lambda / 2 \pi)$, where $D$ is the distance and $\lambda$ is the wavelength) have widely been studied for biomedical implants, wireless sensors and RFID tags [1-4]. The general goal in these techniques is to transfer the data and required energy to a small terminal in a wireless manner. The major advantage of magnetic energy transfer methods is low sensitivity to its surroundings, which is mainly composed of magnetically neutral material, thus not interacting with the coupled magnetic field. The other, rather more important advantage, compared to radiated energy transfer, is that the magnetic fields are of less health concern. Different tissues in human body are all electrically lossy material and dissipate the energy in form of heat when exposed to electric field, but their permeability is unity; therefore the human body does not interact with magnetic fields.

Optimized inductive links have been reported to transfer energy with efficiencies of up to $90 \%$ for very short distances (less than $1-3 \mathrm{~cm}$ ) [5]. However, the efficiency of these inductive links drops significantly for longer ranges (decays as $1 / \mathrm{r} 3$ [6]). In [7-8], the transmitting and receiving circuitry were forced to tune to a resonant frequency. This approach has been proven to be useful for energy transfer in very short ranges.

A wireless non-radiative mid-range $(10-100 \mathrm{~cm})$ energy transfer method based on inductive resonance was suggested in [9-12]. Based on this method, the transmitting and receiving circuitry are designed such that they are tuned to a resonant frequency with high quality factors (Q); therefore the distance between the energy emitter and receiver (D) can be increased to multiples of the device characteristic size $\left(\mathrm{L}_{\mathrm{dev}}\right)$, i.e. $\mathrm{D} \sim \alpha^{*} \mathrm{~L}_{\mathrm{dev}}(1 \leq \alpha \leq 5)$, with an efficiency much higher than that obtained by conventional inductive nonresonant coupling methods [8-10]. This method is used to empower coils of radii of $30 \mathrm{~cm}$ with wire cross section of $3 \mathrm{~mm}$. An efficiency of $40 \%$ for $2 \mathrm{~m}$ distance was achieved. However, the magnetic coupling between the coils and therefore the energy transfer efficiency decreases significantly as the coil sizes shrink, due to the almost linear relationship between the area of the transmitting and receiving coils and the mutual coupling between two coils, M, approximated as [9-11]:

$$
M=\frac{\pi}{4} \mu_{0} N_{1} N_{2} \frac{\left(r_{1} r_{2}\right)^{2}}{D^{3}}
$$

In addition, the resonance quality factor $(\mathrm{Q})$ is proportional to the radius of the coil and the conductor thickness. Therefore, energy transfer efficiency is degraded significantly as the coils become smaller and their wires become thinner.

The focus of this paper is to suggest a systematic design method for wireless energy transfer with optimal efficiency between any pair of inductive coils at medium range (10$100 \mathrm{~cm})$, in particular coils with medium device size. It is shown that for each pair of coils located at a certain separation (D), an optimum energy transfer frequency can be identified based on the measurement or theoretical calculations. In addition to coil specifications such as size, number of turns and core material, the optimum frequency is a function of several parameters such as variations in the distance and alignment of the coils, and load variations. In some cases, more than one optimal frequency may exist.

An estimate of the optimum frequency and energy transfer efficiency are found by measuring the network parameters with respect to frequency and suggested closed-form expressions.

In addition, it is shown that tuning to the optimal energy transfer frequency for each system and impedance matching at the transmit and receive ends, can significantly enhance the energy transfer path gain (up to $6 \mathrm{~dB}$ increase in path gain or an increase by a factor of 4 in the energy transfer efficiency). 
The proposed method has been tested using several low profile prototypes that model typical use case for portable devices. For instance, two pairs of medium size coaxially aligned coil pairs of radius $6 \mathrm{~cm}$ and $12 \mathrm{~cm}$ are used to verify the performance of the suggested methods. Using the methods discussed in this paper, it is shown that for the $12 \mathrm{~cm}$ coil pair, an efficiency of about $25 \%$ can be attained for a distance of $10 \mathrm{~cm}$. This efficiency drops to about $4 \%$ at a distance of $0.5 \mathrm{~m}$. Similarly, measurement results for the $6 \mathrm{~cm}$ coil pair confirm an efficiency of about $16.5 \%$ for about $10 \mathrm{~cm}$ distance that drops to $2.5 \%$ for distance of about $0.5 \mathrm{~m}$.

The structure of this paper is as follows: Section II describes the system modeling and theoretical estimation of the optimum resonance frequency based on coil dimensions and distance according to [11]. Also, measurement-based optimization of the matching circuitry is presented in Section II. In Section III, two sets of coils are used as prototypes to verify the estimated performance. Efficiency enhancement methods are suggested and verified based on optimum frequency tuning and impedance matching at the transmitter and receiver side. Finally, concluding remarks and summary are briefly discussed in Section IV.

\section{SYStem Modeling AND MeASUREMENT APPROACHES}

\section{A. Theoretical Approximation}

The quality factor and resonant frequency of a coil are defined as:

$$
Q=\frac{\omega \cdot L}{\left(R_{\text {ohm }}+R_{\text {rad }}\right)}
$$

and

$$
\omega=\frac{1}{\sqrt{L C}}
$$

respectively, where $L$ is the inductance of the coil, $\mathrm{C}$ is the equivalent parallel capacitance of the coil (including the self capacitance and parasitics) and $R_{\text {ohm }}$ and $R_{\text {rad }}$ are the ohmic loss inside the wire and the radiation resistance (i.e. the energy that is radiated), respectively. The impedance of the wire loop coil with $\mathrm{N}$ turns, and a radius $\mathrm{r}$ conducting wire with circular cross section of radius a, surrounded by air, can be calculated as:

$$
L=\mu_{0} N^{2} r\left[\ln \left(\frac{8 r}{a}\right)-2\right]
$$

Fig.1 shows the optimum frequency of energy transfer based on the method proposed in [10-12] for two sets of coils: (a) the $\mathrm{r}=6 \mathrm{~cm}$ coil pair with $\mathrm{N}=12$ turns and wire cross section radius of $a=0.36 \mathrm{~mm}$, and (b) the $\mathrm{r}=12 \mathrm{~cm}$ coil pair with $\mathrm{N}=6$ turns and wire cross section radius of $\mathrm{a}=1.2 \mathrm{~mm}$.

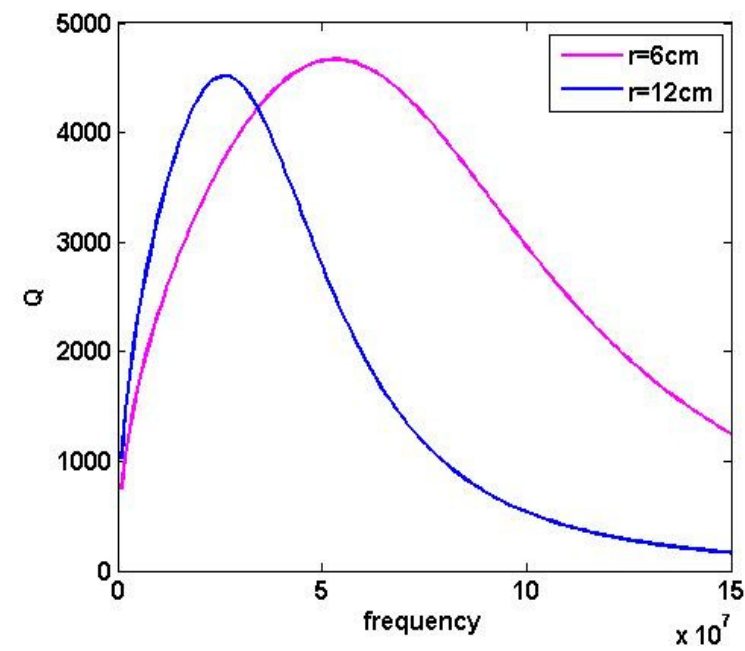

Fig. 1: Estimation of optimum resonant frequency for the $\mathrm{r}=6 \mathrm{~cm}$, and $\mathrm{r}=12 \mathrm{~cm}$ coil pairs using analytical expressions.

It can be seen that the optimal energy transfer frequency (maximum Q) is estimated at around $54 \mathrm{MHz}$ for the $\mathrm{r}=6 \mathrm{~cm}$ coil pair and around $27 \mathrm{MHz}$ for the $\mathrm{r}=12 \mathrm{~cm}$ coil pair. It should be noted that the theoretical predicted values for $\mathrm{f}, \mathrm{Q}$ or efficiency are not accurate due to various reasons, in particular for the smaller set of coils that resonate at a higher frequency. The analytical equations are based on lowfrequency analysis and ignore the dependency of the parameters to the frequency. Several simplifying assumptions are used that can substantially affect the high frequency performance estimation. As an example, the pitch (spacing and angle of two turns of wire in the loop), which makes the loop modeled more appropriately as a helix rather than a loop wire, or the effect of the parasitic capacitances are not accounted for in the equations. Also, in case of modeling as a helix, it is hard to write a closed-form expression, and numerical methods may be required.

Fig. 2 depicts the dependence of $\mathrm{Q}$ and the optimum resonance frequency on (a) the coil radius and (b) the wire cross section radius. As stated before, the resonant frequency increases and the resonance quality factor decreases as the coil size becomes smaller, i.e. with smaller radius or thinner wire or both.

\section{B. Network Parameters}

The resonant frequency, as well as the self and mutual impedance of a coil pair can be characterized using a vector network analyzer (VNA). To do this, the pair of coils can be represented as a two-port network. One possible realization of this two-port network is shown in Fig. 3. In this model, the two coils $\mathrm{L}_{1}$ and $\mathrm{L}_{2}$, located at the desired distance of $\mathrm{D}$ with a mutual inductance of $\mathrm{M}$, are assumed to have total equivalent series losses of $R_{1}$, and $R_{2}$ (including radiation and ohmic losses) and total equivalent parallel capacitances 


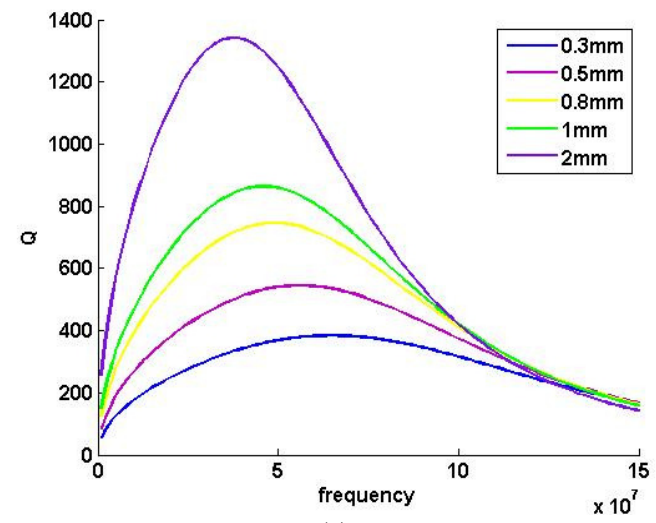

(a)

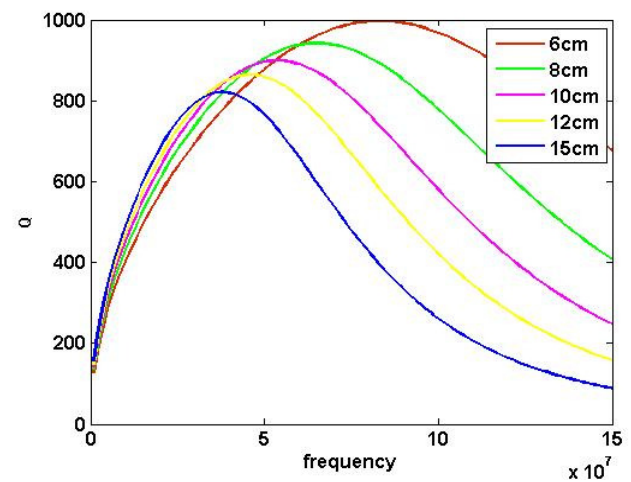

(b)

Fig. 2: Variations of $\mathrm{Q}$ and optimum resonance frequency with (a) the wire radius, a, for $\mathrm{r}=12 \mathrm{~cm}, \mathrm{~N}=1$, and (b) the coil radius, $\mathrm{r}$, for $\mathrm{a}=1 \mathrm{~mm}$, $\mathrm{N}=1$.

of $\mathrm{C}_{1}$ and $\mathrm{C}_{2}$ (including parasitics), respectively. VNA measurements can provide a good approximation of these values at each frequency. Therefore, proper tuning and matching circuitry can be designed to enhance the resonance quality factor and the energy transfer based on these measurements.

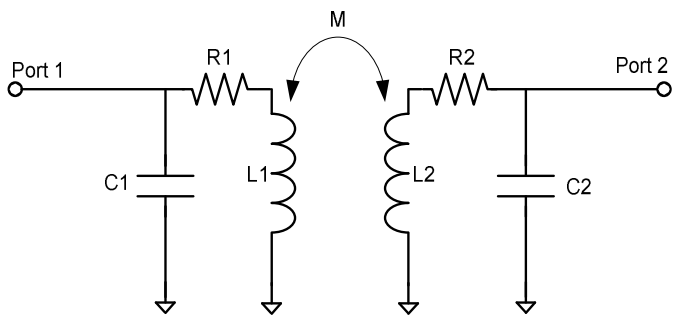

Fig. 3: The two-port Network Model of the coils.

The frequency $f_{i}$ for which the maximum $S_{21}$ is attained is a good approximation of the optimum resonant frequency of the modeled two-port system. However, it should be noted that this maximum value does not necessarily equate the maximum achievable efficiency between the two coils ( $\eta$ ) unless an acceptable impedance matching exists at the two ports, so the return losses are negligibly small. The $S_{11}$ and $\mathrm{S}_{22}$ parameters for this network provide a measure of the impedance matching at the input and output ends, that is, a measure of how much of the provided power by the VNA $\left(\mathrm{P}_{1}\right)$ is received by the transmitting coil $\left(\mathrm{P}_{3}\right)$, and how much of the received power by the receiver coil $\left(\mathrm{P}_{4}\right)$ is transmitted to the VNA port $\left(\mathrm{P}_{2}\right)$ for measurement. Thus the maximum achievable efficiency can be calculated by masking the mismatch losses at both send and receive ends, using Friis equations [6]:

$$
\eta=\frac{1}{\left(1-S_{11}\right)\left(1-S_{22}\right)} \cdot S_{21}
$$

Usually, if $S_{11}$ and $S_{22}$ values less than $-10 \mathrm{~dB}$ are a good approximation of matching. Better resonance $Q$ s can be attained for better matching. For an acceptable matching $\left(\mathrm{S}_{11}, \mathrm{~S}_{22}<<1\right)$, the efficiency equation can be simplified to $\eta=S_{21}$.

It should be noted that at the resonant frequency, the $S_{11}$ and $S_{22}$ values are minimized and the $S_{21}$ and $S_{12}$ (symmetric network) values are maximized. Usually, multiple resonances of the network response can be observed in the measurements. However, the first resonance (lowest frequency) will provide the most efficient energy transfer. Subsequent resonances usually excite multiple modes and the coil has a lower efficiency.

Care should be taken to avoid some systematic errors while measuring with VNA. These inaccuracies might be mainly due to the radiation from feeding cables. This effect can be reduced to some extent by using ferrites. Ferrites will block galvanic coupling of the coils and VNA cables. This is more pronounced when radiated fields are dominant. With dominant near fields (i.e. when lumped components are used for pulling resonance to lower frequencies) the role of ferrites is less significant.

To enhance matching and efficiency results, a matching circuit using lumped elements matching circuitry is designed. A practical system implementation is used to measure the energy transfer efficiency as shown in Fig. 4 (excluding the dashed blocks). On the transmit side, this system is composed of a signal generator, a power amplifier (PA) (together with its biasing circuitry), and a transmitting coil. At the receive side, the system is composed of a receiving coil, and a spectrum analyzer. The spectrum analyzer depicts the received power level at the receiving coil. The PA used

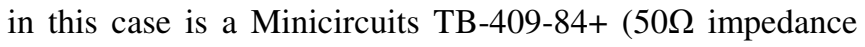
termination) with a gain of $24 \mathrm{~dB}$. An input power of $-24 \mathrm{dBm}$ is applied to the PA, therefore a total of $0 \mathrm{dBm}$ is applied at the transmit side:

Total Tx Power $=($ Psignal gen $)+($ PAGain $)=(-24 \mathrm{dBm})$ $+(24 \mathrm{~dB})=0 \mathrm{dBm}$,

and the path gain equates the received power:

Path Gain $=($ Total Rx Power $)-($ Total $\mathrm{Tx}$ Power $)=$ $($ Total Rx Power $)-(0 \mathrm{dBm})=\mathrm{Rx}$ Power.

Note that:

Path Gain $=10 * \log _{10}($ Efficiency $(\%))$. 
If impedance matching between the transmitting coil and the PA at the transmit side and between the receiving coil and the spectrum analyzer at the receiving side is used (the dashed blocks in Fig. 4), and the transmitting frequency is chosen to be the optimal frequency of energy transfer, a good measure of the energy (power) transfer efficiency (the path gain) can be attained. The optimal frequency can be attained by sweeping the transmitting frequency at the transmit side on a bandwidth around the optimum frequency, and continuously monitoring the gradient of received energy (power) to detect the maximum received energy (power) level at the receive side.

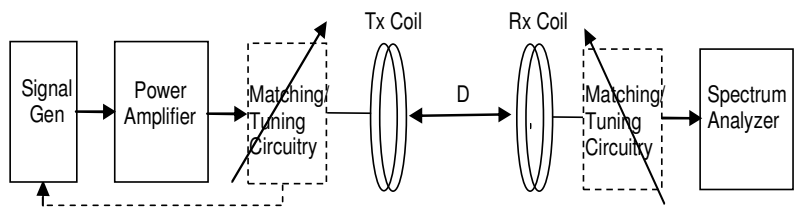

Fig. 4: The prototype setup including the lumped-element matching circuitry.

A feedback path from the adaptive matching/tuning circuitry to the signal generator can therefore be used to optimize the system by automatically locking to the frequency step before the energy transfer gradient becomes negative. The optimal frequency for the setup is very close to that measured by the VNA, but the path gain level is lower than the maximum measured S21 by a VNA. The path gain measured using this method can be considered as the most accurate measurement compared to previous methods. Similar to the case for the VNA, matching and frequency tuning circuitry should be used to enhance the energy transfer efficiency. This will be discussed in detail in the following section.

\section{MEASUREMENT RESULTS AND OPTIMIZATION}

\section{Example 1: The $\mathrm{r}=6 \mathrm{~cm}$ Coil Pair}

\section{A. Network Parameter Measurements}

Fig.5 depicts network parameters for a pair of coaxially aligned circular coils (shown in Fig.6) with a radius of $\mathrm{r}=6 \mathrm{~cm}$ and $\mathrm{N}=10$ turns of a copper wire of radius $\mathrm{a}=0.11 \mathrm{~mm}$ at a distance of $\mathrm{D}=15 \mathrm{~cm}$.

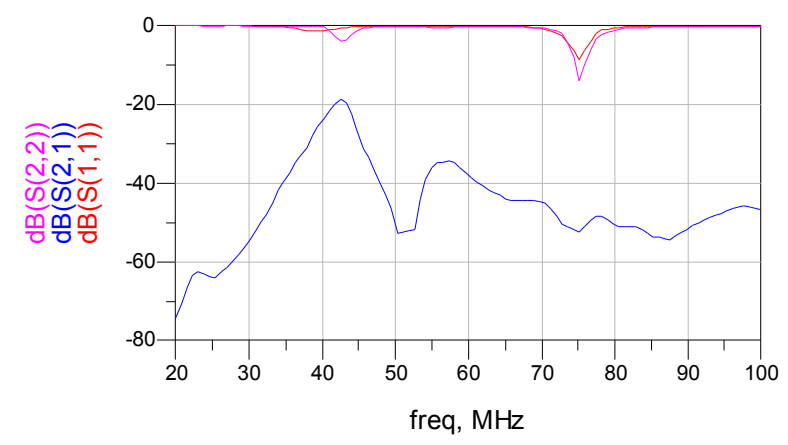

Fig. 5: S-parameters for the pair of $\mathrm{r}=6 \mathrm{~cm}$ coils, before matching, optimum resonant frequency is $42.52 \mathrm{MHz}$.
As can be seen in this figure, optimum resonant frequency is at $42.52 \mathrm{MHz}$ with an $\mathrm{S}_{21}$ of $-18.5 \mathrm{~dB}$. It is evident from Fig. 5 for $S_{11}$ and $S_{22}$ that the two coils are similar but not identical. These measurements were carried out using ferrites at the VNA port ends.

Applying the path gain and $S_{11} / S_{22}$ parameters in equation (5), we can predict the path gain and therefore the efficiency that can be attained after matching to be $16 \%$.

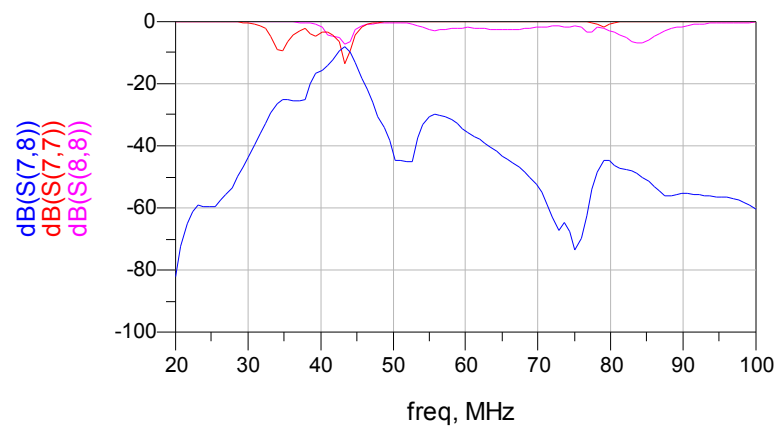

Fig. 6: S-parameters for the pair of $\mathrm{r}=6 \mathrm{~cm}$ coils, after matching, optimum resonant frequency is $43.29 \mathrm{MHz}$.

Fig. 6 depicts the S-parameter results after adding a pair of series matching coils of $300 \mathrm{nH}$. It can be seen that the resonance frequency is slightly shifted by the matching circuitry; however, a path gain of about $-8 \mathrm{~dB}$ can be attained using this matching, that matches well with theory.

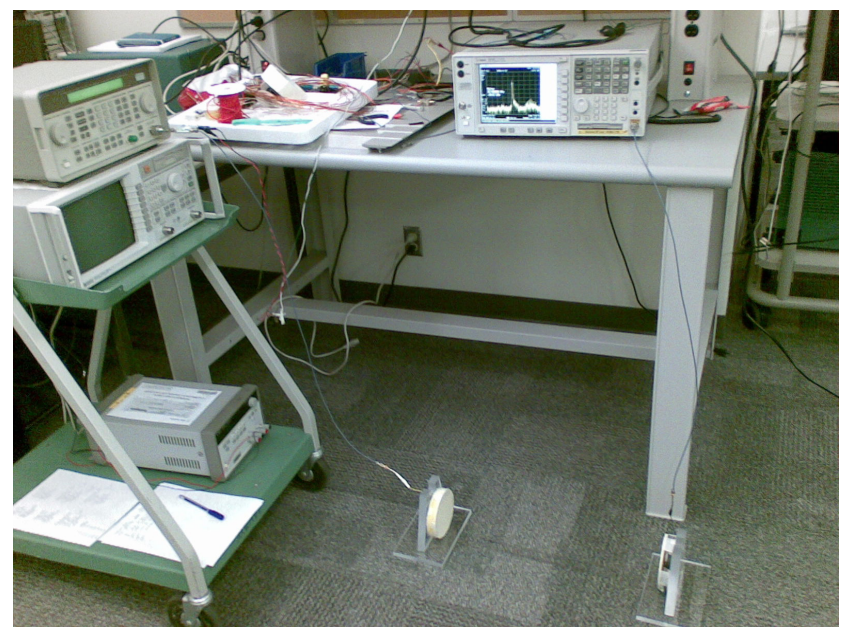

Fig. 7: Picture of the test setup including a spectrum analyzer and a power amplifier. The $6 \mathrm{~cm}$ coils are also shown.

Therefore, impedance matching can be used as an effective method to enhance the energy transfer efficiency in the system. It should be noted that depending on the distance between the two coils, the measured coil parameters and the optimum energy transfer frequency may vary.

\section{B. Practical System Measurements}

To measure the practical efficiency results for this pair of coils, a system setup using a PA and a spectrum analyzer was used (picture shown in Fig 6, including the $6 \mathrm{~cm}$ coils). Fig. 8 (a) and (b) show the measurement results of the setup for the variations of path gain and efficiency versus distance for the 
pair of coils, after matching at the constant energy transfer frequency of $42.4 \mathrm{MHz}$ (blue), and after matching but tuned at the optimum energy transfer frequency for each distance (red).

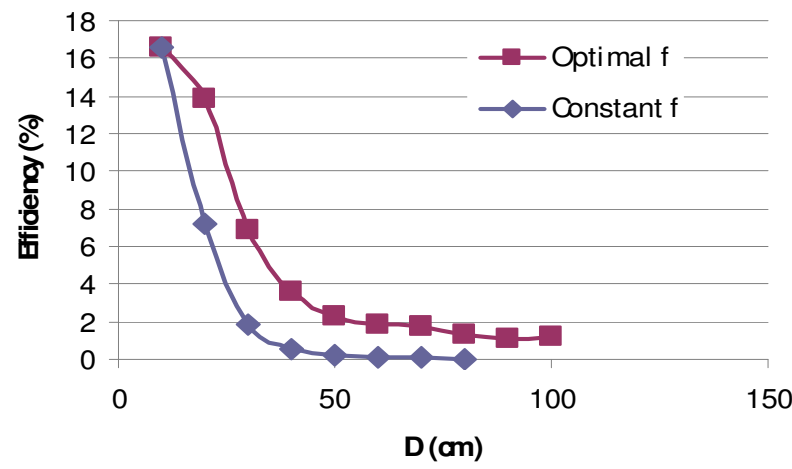

(a)

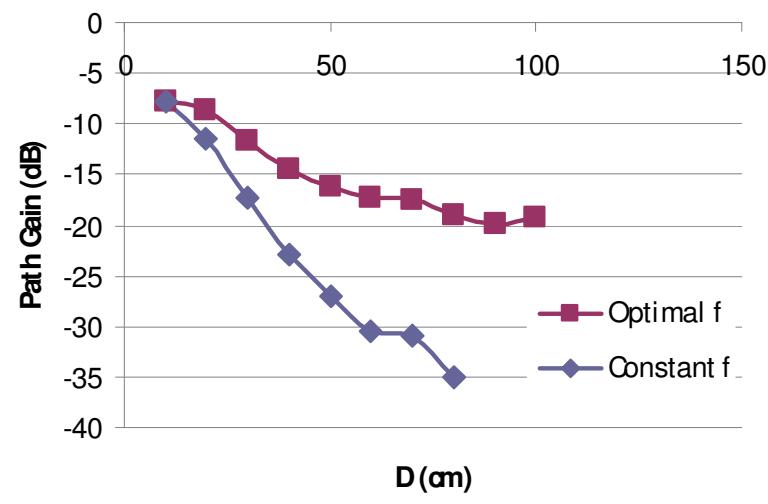

(b)

Fig. 8: Spectrum analyzer measurement results: (a) power transfer efficiency and (b) path gain vs. distance for $\mathrm{r}=6 \mathrm{~cm}$ coil pair at constant frequency (blue) and tuned to optimal frequency (red).

It can be seen that tuning to the optimum frequency (also a form of impedance matching) can almost double the efficiency at a distance of $20 \mathrm{~cm}$ (from $7.2 \%$ to $13.8 \%$ ). Also, it can be seen that for a distance of $50 \mathrm{~cm}$, up to $-16 \mathrm{~dB}$ path gain, equivalent to $2.5 \%$ efficiency can be attained.



Fig. 9: S-parameters for the pair of $\mathrm{r}=12 \mathrm{~cm}$ coils, after matching, optimum resonant frequency is within the $24-30 \mathrm{MHz}$ band.
Note that the efficiency enhancement by tuning to optimal frequency is more efficient in longer ranges. This is because of the fact that the second and first order terms in the radiation equation become more significant. These low order inverse terms decay in a slower rate by distance.

\section{Example 2: The $\mathrm{r}=12 \mathrm{~cm}$ Coil Pair}

\section{A. Network Parameter Measurements}

Similarly, Fig. 9 depicts the network parameter measurement results for the pair of coils with radius of $\mathrm{r}=12 \mathrm{~cm}$ and $\mathrm{N}=4$ turns and $\mathrm{a}=0.11 \mathrm{~mm}$, with $\mathrm{D}=15 \mathrm{~cm}$, after matching. In this case, a band of optimum resonant frequencies is identified from 24 to $30 \mathrm{MHz}$. The designed matching circuit moves the intersection of the impedance curve with the real axis closer to $50 \Omega$ point. However as can be seen, the proximity of two resonances has created a wideband matching with the drawback of reduced quality factor. Wideband operation is beneficial for data transfer applications and to reduce the sensitivity of the system in order to attain a robust energy transfer setup.

A pair of series L-C circuit is designed $(\mathrm{L}=1 \mathrm{uF}, \mathrm{C}=$ $100 \mathrm{pF}$ ) in order to improve the return loss at both ends. This matching circuitry improves the $\mathrm{S}_{21}$ from -11 to $-5.8 \mathrm{~dB}$.

\section{B. Practical System Measurements}

The experimental results for (a) efficiency and (b) path gain vs. distance are shown in Fig. 10, at a constant resonance frequency of $30.55 \mathrm{MHz}$ (optimum resonant frequency for $10 \mathrm{~cm}$ separation between the coils, shown in blue) and tuned to the optimal resonance frequency at that distance (in red). It can be seen that an energy transfer efficiency of about $25 \%$ can be attained after matching at $10 \mathrm{~cm}$. This efficiency drops to about $4 \%$ at a distance of $100 \mathrm{~cm}$. Note that by tuning to the optimum frequency at each distance between the coils, a path gain increase of at least $5 \mathrm{~dB}$ can be attained, which is equivalent to energy transfer efficiency increase by factor of 3 .

\section{CONCLUSIONS}

In this paper, mid-range wireless energy transfer for low profile inductive resonant coils is investigated. It is shown that for each system of two coils with a certain separation, an optimum resonant frequency can be found where the energy transfer efficiency is maximized. The system is modeled as a two-port network and the s-parameters of the system are measured using a VNA. These measurements are then used to estimate the optimum resonant frequency as well as to design the impedance matching circuitry for the coils. Finally, the actual performance of the system is tested using a signal generator and a power amplifier at the transmit side and a spectrum analyzer at the receive side.

Two sets of prototype coils with radius of $6 \mathrm{~cm}$ and $12 \mathrm{~cm}$ are used to verify the suggested methods. It is shown that by tuning to the optimum resonant frequency and designing proper matching circuitry, path gain and energy transfer 
efficiency can be improved significantly. An efficiency of about $25 \%$ for $12 \mathrm{~cm}$ coils and $16.5 \%$ for $6 \mathrm{~cm}$ coils are measured for a distance of $10 \mathrm{~cm}$. This efficiency drops to about $4 \%$ for $12 \mathrm{~cm}$ coils and $2.5 \%$ for $6 \mathrm{~cm}$ coils for a distance of $50 \mathrm{~cm}$. It should be noted that while many of the methods used in this paper may be common practice in RF design, little has been exploited for mid-range wireless energy transfer, in particular based on magnetic resonance.

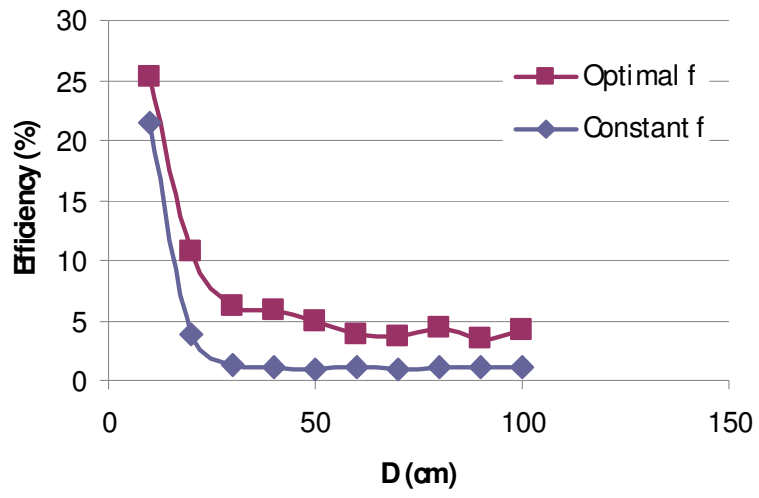

(a)



(b)

Fig. 10: Spectrum analyzer measurement results: (a) power transfer efficiency and (b) path gain vs. distance for $\mathrm{r}=12 \mathrm{~cm}$ coil pair at constant frequency (blue) and tuned to optimal frequency (red).

\section{REFERENCES}

[1] P. V. Nikitin, K. V. S. Rao, and S. Lazar, "An Overview of Near Field UHF RFID,” RFID, IEEE Int. Conf., March 07, pp. 167 - 174.

[2] M. Catrysse, B. Hermans, and R. Puers, "An inductive energy system with integrated bi-directional data-transmission," Elsevier Science Direct, Sensors and Actuators 115 (2004), 221-229.

[3] Y. P. T. Theilmann, and P. M. Asbeck, "An Analytical Model for Inductively Coupled Implantable Biomedical Devices with Ferrite Rods," IEEE Trans. Biomedical Circuits and Systems, Vol. 3, No. 1, Feb. 09.

[4] B. Jiang, J. R. Smith, M. Philipose, Sumit Roy, K. S. Rajan, and A.V. Mamishev, "Energy Scavenging for Inductively Coupled Passive RFID Systems," IMTC 2005 - Instrumentation and Measurement Technology Conf., Ottawa, Canada, May 2005.

[5] G. Vandevoorde, and R. Puers, "Wireless energy transfer for standalone systems: a comparison between low and high energy applicability," Elsevier, Sensors and Actuators A: Physical ,Volume 92, Issues 1-3, Pages 305-311, August 2001.
[6] C. A. Balanis, Antenna Theory: Analysis and Design, $3^{\text {rd }}$ Ed. New York, NY: John Wiley and Sons, 2005.

[7] G. Wang, L. Wentai, M. Sivaprakasam, and G. A. Kendir, "Design and analysis of an adaptive transcutaneous energy telemetry for biomedical implants," IEEE Trans. Circuits and Systems I: Regular Papers, vol.52, no.10, pp. 2109-2117, Oct. 2005.

[8] N. N. Donaldson, and T.A. Perkins, "Analysis of Resonant Coupled Coils in the Design of Radio Frequency Transcutaneous Links," Med. \& Biol. Eng. \& Comput., 1983, 21, pp.612-627.

[9] A. Karalis, J.D. Joannopoulos, and M. Soljacic, "Efficient wireless non-radiative mid-range energy transfer," Elsevier, Annals of Physics 323 (2008), 34-48.

[10] A. Kurs, A. Karalis, R. Moffatt, J. D. Joannopoulos, P. Fisher, and M. Soljacic, "Wireless Energy Transfer via Strongly Coupled Magnetic Resonances," $\quad$ Science $317, \quad 83$ (2007), DOI: 10.1126/science.1143254.

[11] J. D. Joannopoulos, A. Karalis, and M. Soljacic, "Wireless NonRadiative energy transfer," US Patent number: 20070222542.

[12] N. Cook et al., "Wireless Energy Apparatus and Method," US Patent number: 20080211320 